\title{
Homomorphic Filtering of DT-MRI Fields
}

\author{
C.A. Castaño Moraga ${ }^{1}$, C.-F. Westin ${ }^{2}$, and J. Ruiz-Alzola ${ }^{1,2}$ \\ 1 Medical Technology Center, Gran Canaria Dr. Negrín Hospital.
} Department of Signals and Communications. University of Las Palmas de Gran Canaria, Spain. \{ccasmor, jruiz\}@ctm.ulpgc.es -http://www.ctm.ulpgc.es

${ }^{2}$ Brigham and Women's Hospital, Harvard Medical School. Boston. westin@bwh. harvard.edu

\section{Introduction}

DT-MRI fields measure the self-diffusion of water in biological tissue and, hence, tensors from these fields must be symmetric positive definite (PSD). However, due to a number of reasons this is not true for real data. Furthermore, it would be desirable to maintain that condition when tensor data are filtered. In this paper, we propose a homomorphic processing cascade that guarantees PSD tensor output.

\section{Homomorphic Cascade}

Homomorphic systems satisfy a generalized principle of superposition, and they can be decomposed as a cascade of three homomorphic subsystems [1]. This idea can be used to assure PSD tensors at the output of the processing system by using a homomorphic chain with an output subsystem enforcing such a condition.

The input subsystem to the homomorphic cascade computes the (matrix function) positive square root of the tensors. This is a non-linear operation not obeying the principle of superposition. Nevertheless, taking into account that input data is the addition of signal, $\mathrm{s}[\mathrm{n}]$, and noise, $\mathrm{w}[\mathrm{n}]$, the binomial can be expanded as

$$
(x+y)^{r}=\sum_{k=0}^{\infty}\left(\begin{array}{l}
r \\
k
\end{array}\right) x^{k} y^{r-k}, r \in \mathbb{C} \quad\left(\begin{array}{l}
r \\
k
\end{array}\right)=\frac{\prod_{i=0}^{k-1}(x-i)}{k !}
$$

and 1 when $\mathrm{k}=0$.

Expanding the series in $s[n]$ and assuming that noise is small with respect to the signal, the series can be truncated.

$$
\begin{array}{r}
(s[n]+w[n])^{1 / 2}=s[n]^{1 / 2}+\frac{s[n]^{-1 / 2} w[n]}{2}-\frac{s[n]^{-3 / 2} w[n]^{2}}{4} \\
+\frac{3 s[n]^{-5 / 2} w[n]^{3}}{8}-\ldots=\hat{s}[n]+\hat{w}[n]
\end{array}
$$

In this way, the system satisfies the homomorphic property, maintaining the addition as the operation, so that a linear system in the usual sense can be used in the middle of the cascade to process the tensor field. 
Since the input subsystem requires PSD tensors at its input, we set to zero negative eigenvalues and perform a tensor smoothing as a pre-processing stage.

The output subsystem is the inverse of the square root of a tensor. An analogous demonstration can be carried out to justify taking squares as an homomorphic system. Moreover, the output subsystem enforces the resulting tensors to be PSD.

\section{Results and Conclusions}

In order to illustrate the proposed cascade performance, white Gaussian noise is added to a stationary synthetic tensor field (Fig. 1 a), which is filtered using a Wiener filter independently on each component. The output is compared with the original data to obtain an error measure. Fig (1.b) shows the output of the Wiener filter projected on PSD subspace (no homomorphic chain) and Fig (1. c) the homomorphic cascade output, using the Wiener filter in the middle of the chain.

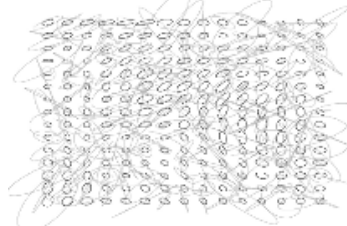

(a) Orig. \& Noisy fields

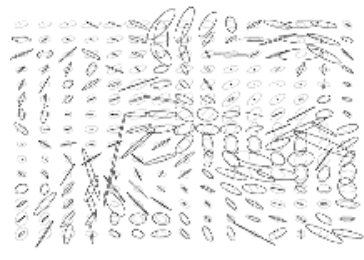

(b) Wiener + PSD

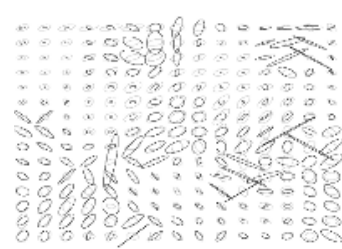

(c) Homom. cascade

Fig. 1. (a) Original (black) and Noisy (gray) fields. (b,c) Original (gray) and filtered (black) fields.

As Fig. (2) shows, filter performance is compared measuring the mean square error (including all the components) as a function of noise power, using a Wiener Filter and PSD projection (gray) and using the proposed Homomorphic cascade (black). The superior performance of our approach is clear from the figure.

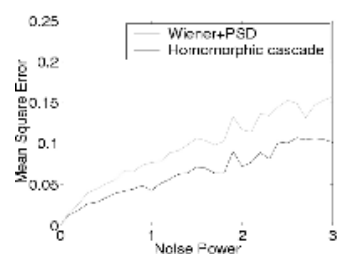

Fig. 2. MSE variation with noise power: Wiener + PSD (gray). Homomorphic cascade (black)

The results demonstrate that the homomorphic cascade performs better than just filtering and projecting the filter output to guarantee PSD tensors, although more validation is still needed and this system has to be tested with real data.

\section{References}

1. A. V. Oppenheim and R. W. Schafer, Discrete-Time Signal Processing, International ed. ISBN: 0-13-216771-9. Prentice-Hall Inc. N.J. 07632, 1989. 